\title{
ARCHITECTURE AND IMPLEMENTATION OF AN INTERACTIVE TOOL FOR THE DESIGN AND VISUALIZATION OF ACTIVE CAPABILITY
}

\author{
Sharma Chakravarthy and Seokwon Yang \\ Computer Science and Engineering Department, The University of Texas at Arlington, \\ Arlington, TX 76109-0015 \\ Email: sharma@cse.uta.edu
}

Abstract: Active capability has been proposed as a paradigm to meet the demands of many database and other applications that require a timely response to situations. Event - Condition - Action (or ECA) rules are used to support active capability in a system. The design of an active DBMS for a particular application entails the design of schema for that application as well as ECA rules that correspond to business rules, constraints, invariants, and situations to be monitored in that application.

This paper concentrates on: i) architecture and development of a generalpurpose lightweight proxy that enables us to support interfaces on distributed or web-based environment uniformly and ii) design and implementation of an interactive visualization tool for Active Database systems (ADBs). The tool is intended both as a debugging tool and a visualization tool based on the proposed architecture. The tool allows the designer/user to set breakpoints on event occurrences and rule execution or to enable/disable events and rules at run time. The priority and other attributes of rules can be changed interactively to study the behavior of rule interaction among themselves and with transaction/applications.

Key words: ECA rules, Event graph, Visualization 


\section{INTRODUCTION}

Active database technology provides a means for database applications to react automatically to user-requested or system-generated events. Specification of this active capability can be tricky requiring new tools for the management of Event-Condition-Action (ECA) rule abstraction. Use of ECA rules for real-life DBMS applications has added a new dimension to the traditional validation or analysis phase. For the traditional DMBS, we used to have only schema design for the application and the main form of validation was to make sure that the design captured all of the requirements of the application and browsing the data returned as a result of user requests. In contrast, in active database systems, we have schema design as well as rule design for an application that uses active capability. The schema design for applications can be done statically with respect to the application semantics; however, the design of ECA rules requires that the rules be validated with respect to transactions and applications, as there is semantic interaction between rules and applications. Furthermore, there is a clear need for regression analysis to make sure additions and deletions of ECA rules does not change the outcome of currently used applications/transactions in an undesirable way. The tool should help the designer understand the impact of changes in rules while rules are added or removed as the business rules change over a period of time in application. For the designer of the rules, the tool will behave as a debugger and a regression analysis tool at a higher level of abstraction as compared to conventional debugging tools (such as $\mathrm{dbx} / \mathrm{xdbx}$ ). Designers are interested in understanding events and rules relevant to a particular transaction/application, order of rule execution, interaction among rules, and potential cycles among the set of rules. The ability to interact with the tool is critical from a designer's viewpoint. On the other hand, for an end user, the actual set of rules executed, the policies enforced for a particular application, and whether policies interact inconsistently are more important than the ability of interaction.

The tool described in this paper is a redesign that not only supports batch mode, but extends it further to on-line and interactive modes as well. In addition, we also have distributed event detection capability (GED) for which the previous tool was not useful. We wanted an architecture that will be useful for debugging distributed applications and can be used from a remote site as well. Hence, the initial portion of this paper discusses the architectural needs and our approach using which visualization as well as monitoring tools could be developed.

The rest of the paper is structured as follows. Section 2 discusses related work. Section 3 elaborates on the design consideration of the visualization tool. Section 4 discusses the web-based architecture of the visual interface 
components that can also support distributed applications. Section 5 describes the implementation details of the visualization tool and termination analysis. Section 6 contains conclusions.

\section{RELATED WORK}

DEAR [1] keeps track of both rules and events. It automatically detects inconsistencies and potential conflicting interactions among rules. But, the approach taken by DEAR works only for primitive events, such as insert, delete, update, and the rules defined over these events. Second, they do not support interactive features that we feel are necessary for a debugger of this sort. The debugging features of PEARD [2] also include detecting potential cycles in rule execution and a utility to examine different rule execution paths from the same point in the rule triggering process. This tool is similar to our visualization tool especially with respect to rule browsing, breakpoint setting, and rule enabling/disabling. But, the approach does not differentiate the external events generated by the applications and the internal events coming from the nested rule execution. SAMOS [3] has several tools, such as a rule analyzer, a rule browser and a rule explanation component. The termination analyzer assists users in checking the termination of rules. One limitation of SAMOS is that it does not differentiate the internal (or nested) event from the external event as it does not support a nested transaction model. A visualization tool was implemented earlier [4] as part of Sentinel that was mostly a post-analysis tool with limited interactive capability.

\section{VISUALIZATION TOOL REQUIREMENTS}

In contrast to a conventional debugging tool, which concentrates on changes to variables, the debugging context of the visualization tool is to verify or validate the correctness of business rule execution with respect to transactions and applications, based on schema and rules specified.

\subsection{General design requirements}

1. Support of different user perspectives: For the rule designer, the tool will behave as a debugger and a regression analysis tool at a higher level of abstraction as compared to conventional debugging tools (such as $\mathrm{dbx} / \mathrm{xdbx}$ ). Rule designers' interests go well beyond a specific application's running behavior of an application. The interactive mode of the tool is critical from the designer's viewpoint. It is also important that as part of the interaction, the designers are able to disable events and rules selectively, and to further indicate what they want to see highlighted (or removed from the display). On the other 
hand, end users are more interested in the application behavior at run time, based on schema and rule design.

2. Portability: Users should be able to use the same tool on multiple platforms and operating systems as well as in a web-based environment (to allow visualization and debugging at locations non-local to the application) uniformly. Scalable architecture should be taken into account to make the tool available in heterogeneous environments.

3. Multiple modes of usage: The tool should provide multiple modes of usage so that a user can choose the mode that is most appropriate for her. The tool at the least needs to support run-time and post-analysis trace modes. In addition, an interactive mode will be helpful to debug a rule set at run time.

4. Multiple visualization: The tool should be able to support visualizations of participating applications in a distributed setting so that the entire distributed application can be debugged and visualized. In addition, multiple visualization of one application is equally important as groups of developers can debug the same application from different perspectives and from different locations.

\subsection{Design Consideration for the Visualization tool}

When we consider the debugging context of sentinel (an ObjectOriented DBMS into which active capability has been incorporated in the form of event-condition-action or ECA rules), we need to take into account the following specific design considerations.

1. Rule and event interaction: The visualization tool is mainly used to help the user to see the interaction among rules and events in an ADBMS. When event(s) occur, the corresponding rules are triggered. And a rule may raise an event, which in turn triggers rules that invoke other events. Without visual aid, this interaction may turn out to be too complicated to analyze and understand.

2. Presentation of nested rule execution to users: In Sentinel (or any active DBMS), rule execution (i.e., condition and action portions of a rule) is done in a sub-transaction. In other words, rules in turn fire other rules in a nested (as cascading) fashion. Sub-transactions can be nested to arbitrary levels and are represented by $n$-ary tree in the visualization tool. The transaction tree grows in a top-down way.

3. User Interaction in application: ADBMS application developers often realize the importance of the debugging tool that is able to provide mechanisms to change the characteristics of rules and events (enable/disable, change in priority, change in coupling mode etc.) at an abstract level. The same tool should allow the user to intervene during an application execution for this purpose.

4. The tool in a Distributed Environment: Allowing multiple instances of the tool to visualize and explore the same rule set simultaneously would be another interesting direction. Starting several (at least two) visualization tools, loaded with same events, but each tool having different set of rules, would be helpful for debugging. Each visualization tool would be responsible for the actual visualization of corresponding rule set, but operations such as breakpoint, disable, enable operation or generating reports, etc, would be performed as needed for each rule set at each display by users/designers. Our proposed architecture will facilitate this without any problem. 


\section{VISUALIZATION TOOL ON THE WEB}

Figure 1 shows two different scenarios where Java user interface interacts with sentinel (or any other) applications. Figure 1-(a) illustrates the typical environment where each application and GUI creates a socket to listen to the incoming message, exchanging information in both directions. Provided that the GUI knows where each applications is executing and applications know where the GUI waits for notifications in some way, applications and GUI can either be executed on the same machine or on different machines. Figure 1-(b) demonstrates the additional requirement

Figure 1 Distributed Environment vs Distributed Web Environment

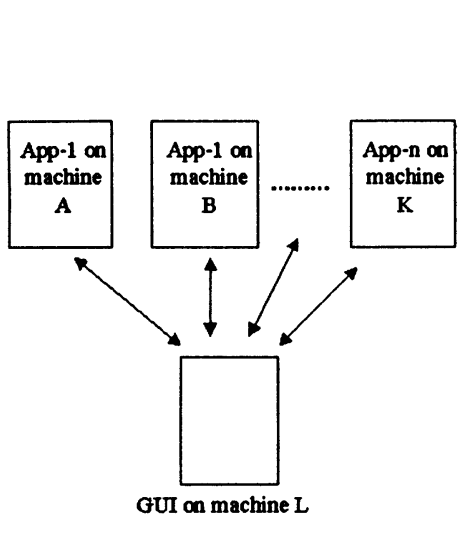

(a) Scenario 1

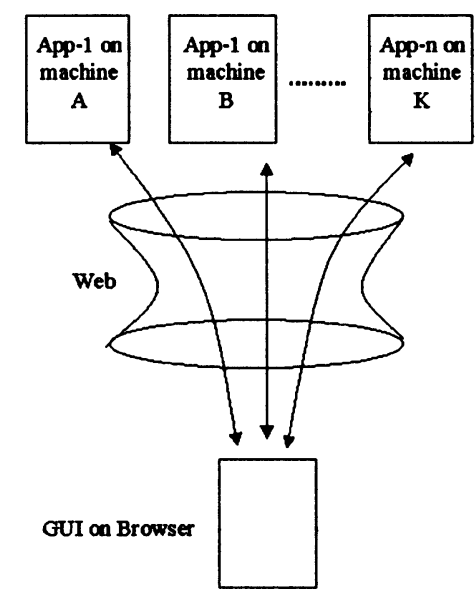

(b) Scenario 2

when we design the GUI components. A GUI should be able to run on top of a browser in which case the communication channel between GUI and applications goes through web. In this context, we define the "Distributed Web environment" as follows. A GUI element runs on a web browser and applications run on remote machines, while bi-directional communication is established through the web server.

The difficulty in reusing the GUI components is related to security restrictions of GUI on top of a browser. In short, the restrictions are as follows:

1. Current commercial browsers do not allow the downloaded GUI components to open a listening port for accepting connections. This restriction comes from the fact that Java applets run on a virtual machine in the browser, which insulates them from direct contact with the host system. This so-called 'sand-box' around the applet enforces restrictions that prevent it from interfering with the host.

2. Messages can only be received after initiating a connection or interaction with the host web server. GUI components on top of a browser cannot create a server 
socket as it is normally done. It cannot receive information from remote applications without initiating a request. It cannot play a role as a server and wait for incoming messages.

3. GUI components are not allowed to open network connections to any computer, other than the web server machine that provided the class files [5].

We use a 3-tier architecture to overcome the above restrictions and create a lightweight proxy. The proxy maintains all connections after the GUI components initialize and register themselves. The information from remote applications to GUI can be transferred through these maintained connections. The user request from GUI to remote applications running on other than web server can be routed through the proxy.

In addition to these security restrictions, we encountered another proxy requirement in the web-based environment. We have Java applets as a frontend interface running on the web browser. One of these GUI components' tasks is to launch the Sentinel (or any general) applications either on the web server machine or a remote machine. In other words, we want to have process control mechanisms similar to that of a UNIX terminal using a browser. For this purpose, we have to add an additional capability in the proxy to receive remote execution requests through the web channel from clients and launch specific processes on the remote machine.

We consider two alternative architectures to implement the proxy. The first architecture uses the fork, semaphores, and shared memory. The other one uses threads and one linked-list for storing the open socket connection. Figure 2-(a) shows the first approach. The proxy creates a child process for each client. It has one global semaphore to coordinate the child processes and one inter-process shared memory to transfer messages received by one child process to another child process. Initially, the child process is in wait state. When any child process receives a message from the client, it first put the message in the shared memory, wakes up the other child process that serves the other client, using the semaphore operation. At the same time, the awakened child process accesses the shared memory, accesses the message and sends the message to the client it serves. Again, the state of child process is also changed into wait state after it sends the message to the destination. 


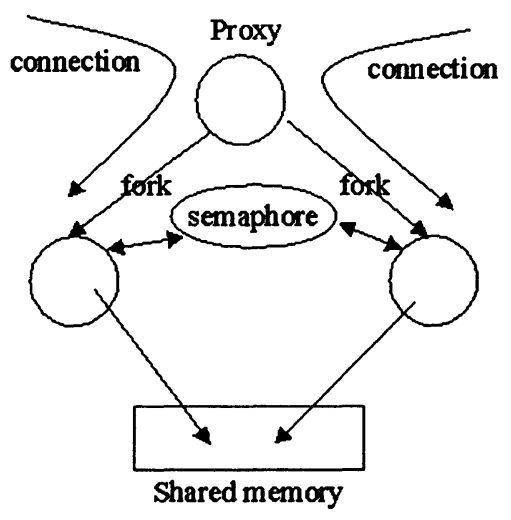

(a)

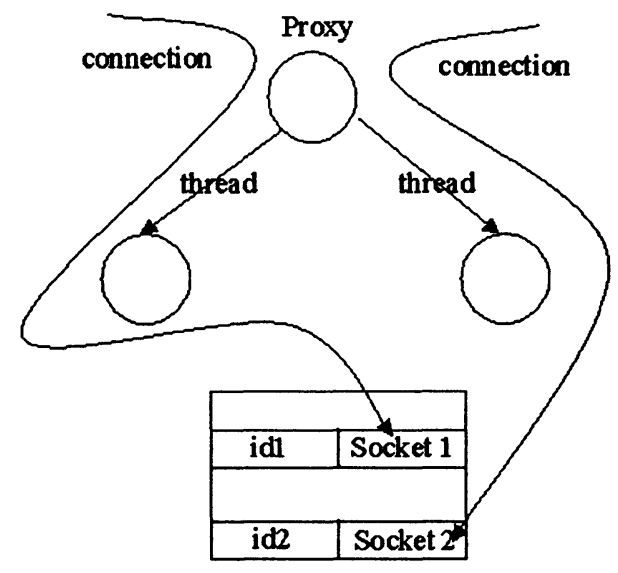

(b)

Figure 2 Two Alternatives of Proxy Architecture

The second approach is shown in Figure 2-(b). When a proxy receives the registering request for connection, it creates a daemon thread to serve each client and puts the socket connection with a unique ID into a global list. The proxy does not close the socket connection that is on the list until the client explicitly asks to close it or the client closes the other end of the socket connection. Each daemon thread is given access privilege to look up the socket connection with a given $\mathrm{ID}$. When a daemon thread receives the request for transferring a message, it first looks up the list to find the destination socket connection with $\mathrm{ID}$, which is contained in the message, and write the message into that socket connection. This will wake up the client and the client processes the message according to the semantics.

We chose the second approach, due to the several limitations of the first approach. One is related to shared memory. Each machine defines a minimum allocation size for shared memory. If the size of shard memory requested does not match the minimum allocation size, the shared memory would not be granted. In some cases, a machine may not have any more shared memory to grant. The other limitation is the number of semaphores required. The first approach uses a semaphore to coordinate the child processes. The number of semaphores needed is determined at run time. Therefore, we cannot make a general-purpose proxy using this architecture. Besides, the first approach cannot port directly to an NT operating system. The resources such as semaphore, shared memory and fork system calls are all specific to an operating system. Also, the size of a process is also large compared to a thread and furthermore process switching is more expensive. 


\section{IMPLEMENTATION OF THE INTERACTIVE VISUALIZATION TOOL}

Sentinel [6] [7] is an integrated active DBMS incorporating ECA rules using Open OODB Toolkit from Tex Instruments [8]. Sentinel allows users to specify events and rules at an abstract level using Snoop [9] language, which is incorporated into the $\mathrm{C}++$ language. Any method of an object class can be a potential event generator (in our case, primitive Event).

Event are classified into i) primitive event which are pre-defined in the system and ii) composite events that are formed by applying a set of operators to primitive and composite events. Primitive events correspond to database operation, such as retrieve, insert, update and delete in RDBMS or method invocation in OODB. Composite events are formed by applying a set of operators to primitive and composite events, recursively. The operators consist of OR, AND, ANY, Seq, Not, Aperiodic Operator, and Periodic Operator. Periodic and aperiodic operators were introduced to meet the requirements of process control, network management, and CIM applications.

Sentinel uses an event tree for each composite event. The trees are merged to form an event graph to detect a set of composite events. Event propagation is performed in a bottom-up fashion. Leaf nodes in event graph correspond to primitive or external events. Internal nodes correspond to event sub-expressions. Each node has a list of subscribers to whom it has to notify once the event denoted by that node is detected.

A sequence of primitive event occurrences (over a period of time) may make a composite occur which is detected in LED. Hence, the local event detector needs to record the occurrence of each event and save its parameters so that they can be used to compute the parameter set of the composite event.

Rules are specified at class definition time as a part of an application. The class-level rule specification is pre-processed into $\mathrm{C}++$ statements and inserted in the application program. Sentinel also support rule activation and deactivation at run time. Rules are specified with a priority. A scheduler, based on the rule priority, controls the execution of the rule thread. If several rules have the same priority, we have concurrent execution of the rules. This may result in the conflict to access data. To deal with this problem, the nested transaction model executes a rule thread as a sub-transaction and uses the transaction synchronization scheme. The order of rule execution can be varied according to the relative priority of its sibling rules.

\subsection{Visual Analysis of ECA Rules}

We have extended the visualization tool developed for Sentinel to enable user interaction at run time. Interaction through a two-way 
communication channel, rather than passively receiving information, allows the user to make changes (enable, disable) on the rule set at run time. The user can set breakpoints during a debugging session so that the state of current rule/event execution can be inspected, and enable/disable rules or events at run time. Other debugging features include a utility for detecting potential cycles in rule execution and a utility to examine different paths from the same point in the rule triggering process [2].

The tool supports 3 modes of operation: post-analysis, real time analysis, and interactive analysis. The difference between the modes is how the rule execution information is supplied to the tool at run time and whether the mode accepts any user interaction during run time. Post-analysis does not allow any interaction while the user application runs. Instead, the LED and transaction management module writes event occurrences and rules that are fired into a $\log$ file as they happen. The visualization tool reads the log file through the proxy server, and simulates the event occurrence and rule execution. Besides, the user can select either step mode or continuous mode with post-analysis mode [10].

Real-time analysis is used to show the changes in active database application as it happens. But, Real time analysis does not allow any user interaction while the applications executes. While the tool and the user application runs, the event occurrences and rule executions, detected by LED, are sent to the tool through proxy using socket connection. When the tool receives the message, the tool parses the message and draws the corresponding action on canvas.

Interactive-analysis mode allows the user to intervene during application execution. The user, typically, selects this mode to set breakpoints during a debugging session so that the state of current rule/event can be inspected. In addition, the user has a choice to enable/disable rules or events at breakpoints.

The visualization tool reads the static information about event trees and rule definitions, which is generated by spp (sentinel pre-processor) when the application is compiled, constructs the event graph on the canvas, and stores the event and rule information in memory (Figure 3). The run time information such as event occurrence and rule execution is supplied in the form of a log file or socket connection according to the 3 different modes we have discussed. The data structure that captures the nested rule execution is an n-nary root transaction is called the top-level transaction and all others are called sub-transactions. The transaction tree grows in a top-down way: It starts from the top-level transaction and spans to descendents.

The transaction manager generates the transaction $\mathrm{ID}$, which is used to infer the parent and child relationship among transactions. The naming is a general-purpose one and accommodates multiple levels and multiple subtransaction. Figure 4 shows an execution snapshot of the visualization tool. It 
shows the composite AND event on stock_e1 and stock_e2 and sequence event on the AND event and stock_e_get_price defined on CLASS stock. It also present the nested rule execution in n-ary tree, comparing with the event graph. The numbers below the boxes indicate the transaction $\mathrm{ID}$, from which the tool knows the nested rule level.

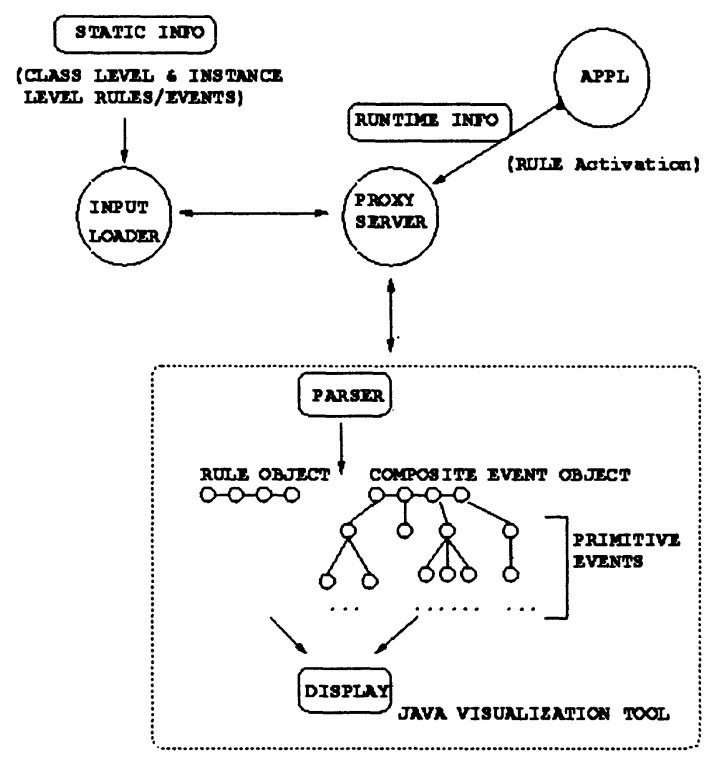

Figure 3 Input to the Visualization Tool

When an event occurs, the visualization tool only changes the color of the event node on the canvas. The user can know from this color change that the event has occurred. When a rule is actually scheduled, LED sends a message to the visualization tool with its sub-transaction $\mathrm{ID}$, and the visualization tool determines the position where the sub-transaction will be drawn from the ID. LED also includes the event ID or event name in Rule execution output so that it can display the relationship between subtransaction and the triggering event.

Coupling mode specifies the point of time after the event occurrence when condition evaluation and action execution begin. To visualize the deferred rule execution separately from the immediate rule execution, the OODB_commitT primitive event is shown as an event in the visualization. While the immediate rules are connected to the triggering event, the deferred rules are connected to the OODB_commitT primitive event when they are schedule. In this way, we can display the semantics of the deferred rule. 
In order to interactively set breakpoints and enable/disable rules, the underlying system needs to support that capability. It is also necessary that events (primitive or composite) are not propagated when there are no rules associated with them. Otherwise, these events will be sent to the visualization tool which will display their detection and propagation. Also, it should be independently possible through the visualization tool to disable events (primitive or composite) even if they have rules associated with them. This is accomplished using a hash table and counters to detect the presence or absence of rules. We define the semantics of disabling a rule as follows.

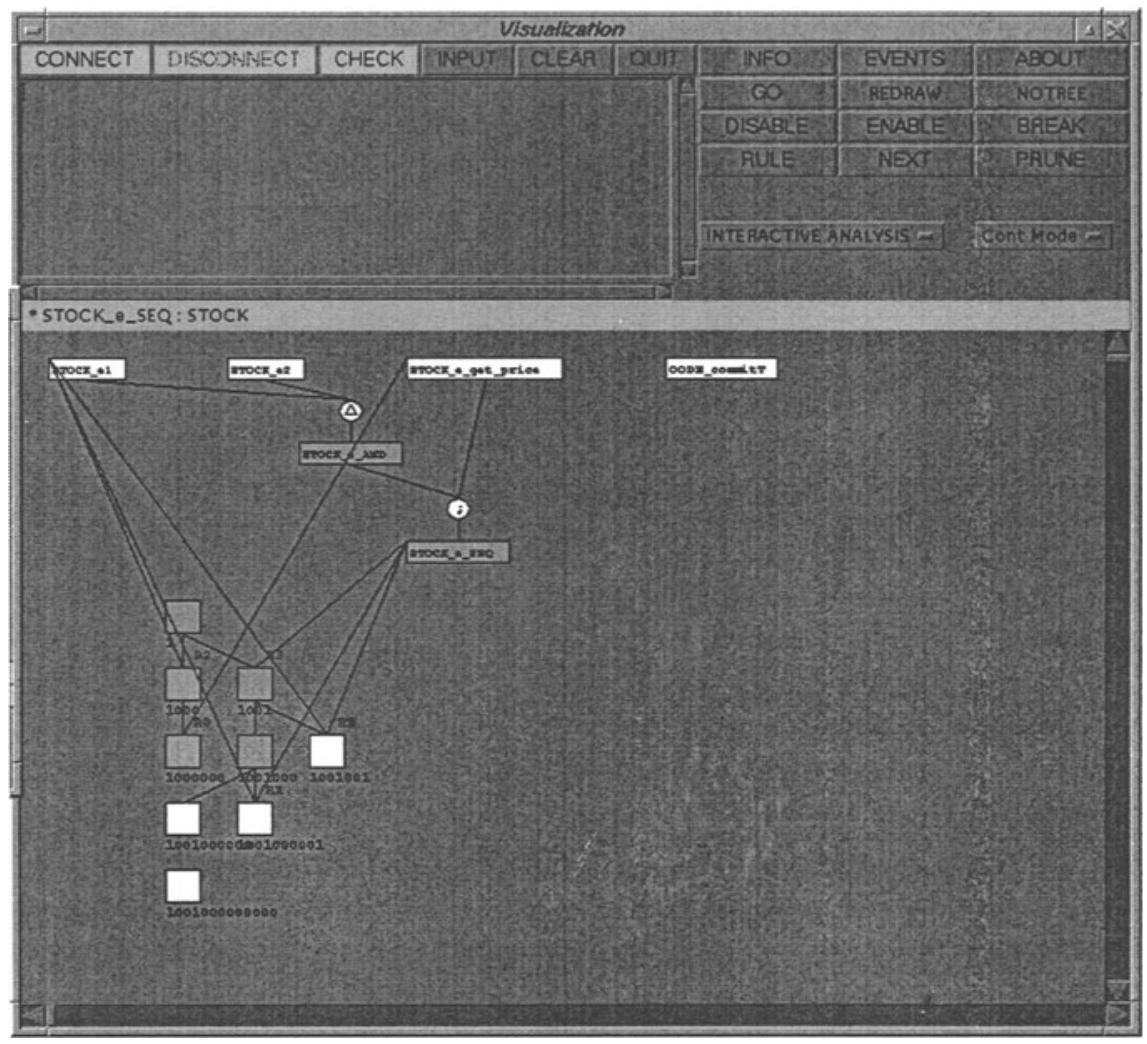

Figure 4 Execution Snapshot of the Visualization Tool

When all the rules subscribed to a particular event are disabled, then LED will stop detecting the event. This is one of the many performance optimizations that have been incorporated into the Sentinel system. This is done at run time.

\subsection{Termination Analysis}

The visualization tool is also extended to find potential cycles that could occur during the execution of an application and identify the rule subset 
potentially leading to an infinite loop. This information helps the rule designer to find the cycle within the narrow scope of rules, instead of going over all defined rules.

\subsubsection{Non-deterministic behavior in rule execution}

The difficulty in performing termination analysis statically is due to the dynamic behavior of rules. The condition evaluation can vary according to the Active DB state, and its execution may change the database state. This dynamic behavior becomes more complicated when we consider the priority and rule-scheduling schemes. Rules may behave differently when scheduled in different orders, yielding unexpected results. Suppose we have two rules when an event occurs. We assume that the execution of the first rule will change the database state into a state, which will make the second rule's condition true. If we execute the second rule first, both rules may not get executed. But, in the reverse order, both rules would be triggered. The same discussion applies to the priorities among rules.

In addition, the rule behavior is very closely related to the semantics of the associated event, and the behavior of event propagation. Figure 5 shows an example of how these factors affect the termination analysis. Event tree shown in both diagrams consists of e1, e2, AND, and sequence events. Event e 3 occurs by the execution of rule $\mathrm{R} 2$. The time when each event occurs is also shown as $\mathbf{t}_{\mathbf{i}}$. Also, $\mathbf{t}_{\mathbf{k}}$ is assumed to be greater than $\mathbf{t}_{\mathbf{j}}$ if is greater than $\mathbf{j}$. As the semantics of sequence event is to detect the event when left child event occurs before the right child event, LED will not detect the sequence event in Figure 5-(a). As a result, R3 will not be fired in Figure-(a). As a result, there is no cycle in the graph in the first diagram. But, in the similar event tree in Figure 5-(b), the sequence event occurs repeatedly and fires rule $\mathrm{R} 3$.

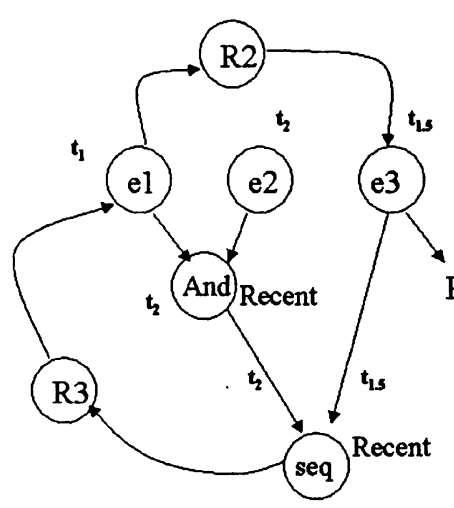

(a)

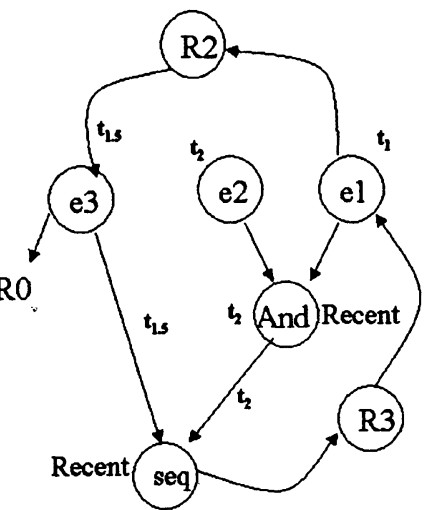

(b)

Figure 5 Cyclic Rule Set Example 
If we consider the dynamic behavior according to context, the problem becomes more complicated. For instance, we may have a different result if $\mathrm{R} 3$ is created in a context other than RECENT.

Another difficulty is that the rule execution may change the database state in such a way that some cyclic rules may stop at some point. This can only be determined at run time.. For example, suppose we have a rule whose action is to deduct $\$ 100$ from saving account only when saving account has more than $\$ 100$. If this rule is part of a cycle, then the cycle eventually stops.

\subsubsection{Our approach}

We consider two approaches to this problem, in our case. One is a passive approach, similar to a conventional programming debugger. The debugger just displays the execution trace and lets the user infer the details of the problem. The debugger does not find the rule set that is potentially cyclic. Refer to [11] for further details of this approach. We shall describe the second approach here. The second one is an active approach, which displays the cyclic rules graphically to users at run time to help users visualize the nature of the problem.

\subsubsection{Visualization of the Cyclic Rules}

The tool would be user-friendly if it is able to show the cyclic behavior to users at run time, in the same way it shows the rule execution and event occurrence. Besides, the stack frame approach has a limitation. It needs an arbitrary numbers of stack frames to be grouped to detect repeating patterns. For example, Figure 6 shows 3 simple different cyclic rules and the change of their stack frames at run time. Note that the cyclic rule path length determines the number of stack frames to identify the repeated pattern. Of course, the examples in Figure 6 are simple cases so it is easy to identify the groups. But, in the real applications, which also have composite events and concurrent rule execution, this grouping is not easy for users.
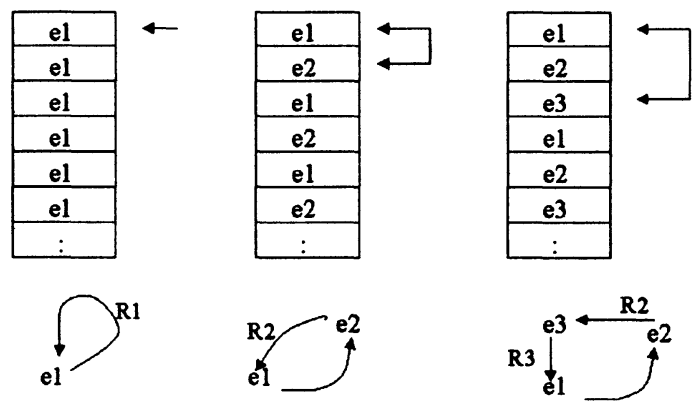

Figure 6 Grouping stacks to identify the repeated patterns 
Figure 7 shows one of ways visualizing cyclic rules to users. Each event

Figure 7 Visualization of

Cyclic Rules as Types

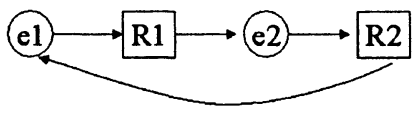

Figure 8. Visualization of cyclic rules in instance level

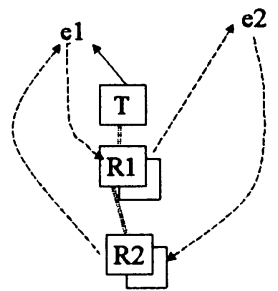

and rule is treated as a type. It does not show the instance level of event occurrence and rule firing. The graph can be generated statically, but needs to be verified at run time. The visualization tool currently generates event graph statically from event definition and displays the rule execution at the instance level to visualize the nested execution of rules. The edges between events and rules are generated at run time.

Figure 8 shows the visualization of cyclic rules at the instance level, which overcomes the disadvantages of the display scheme in Figure 7. Instead of displaying the n-ary tree, the tool positions the same rules at different transaction level side by side. In other words, when the tool detects the same rule execution at different transaction level, the tool assumes a cyclic mode, which is differentiated from display mode of rule execution as a transaction hierarchy explained earlier. To visualize cyclic rules such as the one shown in Figure 7, the tool should be able to identify the rule that generates a certain event and to identify the event that triggers certain rules. In other words, to draw the edge between $\mathrm{R} 1$ and $\mathrm{e} 2$ and edge between $\mathrm{e} 2$ and $\mathrm{R} 2$ in Figure 7, the tool should be able to identify, from the run time trace, which event is generated by the execution of a rule and which rule is triggered by an event. The tool would obtain this information from LED and draw direct edges between events and rules in the above graph at run time. Current implementation generates messages for the second case. From the message "Rule RULE_NAME O_ID EVENT_NAME", the tool can find the identity of the event that triggers a rule. In addition, the information to draw an edge from a rule to an event also can be easily obtained from LED by generating the current transaction ID with event occurrence. From the transaction $\mathrm{ID}$, the tool is able to know which rule generates an event and show it graphically. 


\section{CONCLUSION AND FUTURE WORK}

This work significantly extends the previous visualization tool developed for Sentinel. The tool has been changed to incorporate the nested sub-transaction model, and extended to have user interaction at run time. The functionality includes the setting of breakpoints, disabling/enabling events and rules. The work also covers the passive cycle detection mechanism to interrupt the application execution when they show the repeated stack frame and static cycle in ETG. Besides, this paper presents a general way to extend active capability to the web-based distributed environment. The work extends the previous 3-tier architecture for designing a proxy to make webbased GUI possible. The redesigning of proxy has considered the portability and making remote process invocation possible to set up the communication among processes. For more details refer to [11].

\section{BIBLIOGRAPHY}

1. O. Diaz, A.J., and N.W. Paton. DEAR: A Debugger for Active rules in an Object Oriented Context. in 1st International Conference on Rules in Database Systems. September 1993.

2. Alexander, S.D. Urban, and S.W. Dietrich, PEARD: A Prototype Environment for Active Rule Debugging. Intelligent Information Systems : Integrating Artificial Intelligence and Database Technologies, 1996. 7(2).

3. Anca Vaduva, s.G., and Klaus R. Dittrich. Investigating Rule Termination in Active Database Systems with Expressive Rule Languages. in 3rd International Workshop on rules in Database Systems (RIDS 97). June 1997, Sweden.

4. Chakravarthy, S., Z. Tamizuddin, and J. Zhou, SIEVE: An Interactive Visualization and Explanation Tool for active Databases, in Proc. of the 2nd International Workshop on Rules in Database Systems 1995. p. 179--191.

5. Gosling, J. and H. McGilton, The Java Language Environment: 1996.

6. Anwar, E., L. Maugis, and S. Chakravarthy, A New Perspective on Rule Support for Object-Oriented Databases, in 1993 ACM SIGMOD Conf. on Management of Data. 1993: Washington D.C. p. 99-108.

7. Chakravarthy, S., et al., Design of Sentinel: An Object-Oriented DBMS with Event-Based Rules. Information and Software Tech., 1994. 36(9): p. 559--568.

8. David, L.W., A.B. Jose, and W.T. Craig, \{Architecture of and Open ObjectOriented Database Management System\}. IEEE Computer, 1992: p. 74--81.

9. Chakravarthy, S. and D. Mishra, Snoop: An Expressive Event Specification Language for Active Databases. D\&KE, 1994. 14(10): p. 1--26.

10. Han, S., Three-Tire Architecture for Sentinel Applications and Tools: Separating Presentation from Functionality. 1997, Univ. of Florida: Gainesville.

11. Yang, S., Interactive Visualization Tool for Supporting ECA Rule Design, in MS Thesis. 1999, CISE Department, Univ. of Florida: Gainesville. 